\title{
Shell Structure in the Fornax Dwarf Spheroidal Galaxy
}

\author{
Matthew Coleman \\ Research School of Astronomy \& Astrophysics, Institute of Advanced Studies, The \\ Australian National University, Cotter Road, Weston Creek, ACT 2611, Australia \\ coleman@mso.anu .edu . au \\ G. S. Da Costa \\ Research School of Astronomy \& Astrophysics, Institute of Advanced Studies, The \\ Australian National University, Cotter Road, Weston Creek, ACT 2611, Australia \\ gdc@mso.anu .edu . au \\ Joss Bland-Hawthorn \\ Anglo-Australian Observatory, PO Box 296, Epping, NSW 2121, Australia \\ jbh@aaoepp.aao.gov.au \\ David Martínez-Delgado \\ Max-Planck-Institut für Astronomie, Königstuhl 17, D-69117 Heidelberg, Germany \\ ddelgado@mpia-hd.mpg.de
}

Kenneth C. Freeman

Research School of Astronomy and Astrophysics, Mount Stromlo Observatory, Cotter

Road, Weston Creek, ACT 2611, Australia

kcf@mso.anu.edu .au

and

David Malin 
Anglo-Australian Observatory, PO Box 296, Epping, NSW 2121, Australia dfm@aaoepp .aao.gov .au

Received _ ; accepted _

Version of 5 November, 2003 


\begin{abstract}
We present initial results from a wide field photometric survey of the Fornax dwarf spheroidal (dSph) galaxy. The focus here is on a small overdensity of stars located approximately $17^{\prime}$ (670 pc in projection) from the centre of Fornax. Based on imaging data in both $V$ and $I$ bands down to $V \approx 21$, the dimensions of the feature are $\sim 1.7^{\prime} \times 3.2^{\prime}(68 \times 128 \mathrm{pc})$ with an average surface brightness of $25.8 \mathrm{mag} / \operatorname{arcsec}^{2}$ in $V$. A follow-up analysis using the deep $B$ and $R$ band data obtained by Stetson, Hesser, \& Smecker-Hane (1998) indicates that the overdensity is apparently dominated by a relatively young stellar population with an age $\approx 2$ Gyr, though the current data do not rule out the presence of older stars. Our preferred interpretation is that the overdensity represents shell structure in the Fornax dSph galaxy, a phenomenon previously unseen in dwarf galaxies. The shell may be the remnant of a merger with Fornax of a smaller, gas-rich system that occured approximately 2 Gyr ago. The comparitively recent star formation seen in some dSph and $\mathrm{dE}$ galaxies may then also be the result of infall of small relatively gas-rich systems.
\end{abstract}

Subject headings: galaxies: dwarf — galaxies: individual (Fornax) — galaxies: photometry - galaxies: stellar content — galaxies: interactions — Galaxy: halo - Local Group

\title{
1. Introduction
}

Dwarf spheroidal (dSph) galaxies provide important clues to our understanding of the universe. For example, the high mass-to-light ratios found in these systems make them excellent laboratories for the study of dark matter. Further, due to the relatively 
large number of dwarf galaxies, they are useful tracers of matter (both luminous and dark) in the local universe. At present, the most widely accepted model describing the formation of structure in the universe is the Cold Dark Matter (CDM) paradigm, based on hypothetical non-baryonic particles (Peebles 1993; Peacock 1999). CDM predicts that relatively small-scale clumps of dark matter formed from fluctuations in the density spectrum of the early universe. Large structures were then formed through a process of hierarchical merging. According to this theory, the initial dark matter haloes had masses of $\sim 10^{7} \mathrm{M}_{\odot}$ (Mayer et al. 2002; Moore et al. 1999), somewhat smaller than the masses of Galactic dSphs $\left(2 \times 10^{7} \mathrm{M}_{\odot}\right.$ to $7 \times 10^{7} \mathrm{M}_{\odot}$, e.g. Mateo (1998)). Consequently, the inference from the simulations is that the current Galactic dSphs have also formed from accretion events in the early Universe. However, high resolution simulations of individual dark matter halos (Power 2003, personal communication) indicate that late infall is negligible in typical dwarf halos. Therefore, discounting a fleeting encounter with a dark clump in the Galactic halo, dwarf galaxies are not expected to show any significant substructure. Nevertheless, substructure is observed in at least one Galactic dSph, namely Ursa Minor (e.g. Olszewski \& Aaronson (1985); Martínez-Delgado et al. (2001); Bellazzini et al. (2002); Palma et al. (2003); Kleyna et al. (2003)), though in this particular case the origin of the substructure may lie with the tidal field of the Galaxy.

Although CDM accurately reproduces the large-scale structure of the universe, its predictions do not match observations of small scale regions with a high contrast in density. For example, the mass distribution of low surface brightness (LSB) galaxies inferred from their rotation curves do not have the steep density cusp at the core expected from CDM models (e.g. Weldrake, de Blok, \& Walter (2003)). In addition, the number of dwarf galaxies surrounding our Galaxy is an order of magnitude smaller than the mass spectrum predicted by CDM (e.g. Moore et al. (1999)). Further, in the CDM picture, the Galaxy's halo was built up through mergers of low-mass systems such as dwarf galaxies. However, 
the abundance patterns observed in present day dwarfs appear to be distinct from those exhibited by Galactic halo field stars (Shetrone et al. 2001; Tolstoy et al. 2003). Indeed, age, chemical and dynamical inconsistencies make currently existing dwarfs unlikely to be the major building blocks of large systems such as spiral and elliptical galaxies (e.g. Tosi (2003)).

The Fornax dwarf spheroidal galaxy is the brightest object of this type in the Galactic halo $\left(M_{V}=-13.2\right.$, from Mateo (1998)). It lies at a distance of $138 \pm 8 \mathrm{kpc}$, and has a tidal radius of $r_{t}=71^{\prime} \pm 4^{\prime}$ (Irwin \& Hatzidimitriou 1995) equivalent to $r_{t}=2.85 \pm 0.16$ kpc. Fornax is one of nine known dwarf spheroidal companions of the Milky Way, all of which show little or no indication of HI or HII (with the possible exception of Sculptor, see Carignan et al. (1998); Bouchard et al. (2003)). Fornax displays a very complex star formation history, beginning at least 12 Gyr ago and extending intermittently until $\sim 500$ Myr ago (see Stetson, Hesser, \& Smecker-Hane (1998); Saviane, Held, \& Bertelli (2000) and references therein). There are also indications, in the form of an excess of stars beyond the 'tidal radius' of the best-fit King model, that the gravitational potential of the Galaxy is tidally heating the outer regions of this dSph (Irwin \& Hatzidimitriou 1995). However, Walcher et al. (2003) find no such excess, albeit for a larger 'tidal radius' $\left(r_{t}=98^{\prime}\right)$ than found by Irwin \& Hatzidimitriou (1995). Possible asymmetries in the distribution of stars in the inner regions of Fornax have been reported by Hodge (1961), Eskridge (1988) and Stetson, Hesser, \& Smecker-Hane (1998). The latter authors drew particular attention to the difference in distribution between the youngest stars and that for the bulk of the population. A measurement of the proper motion of Fornax, made using Hubble Space Telescope data, indicates that this dwarf is currently at or near perigalacticon (Piatek et al. 2002).

In this paper we examine an overdensity of stars situated approximately $17^{\prime}$ from the 
centre of the Fornax dSph. This overdensity does not correspond to any previously reported asymmetry in the distribution of Fornax stars. By collecting accurate photometry down to the red clump at $V=21$, we have been able to determine the surface brightness and dimensions of this feature. Subsequent analysis of the deep survey data of Fornax provided by Stetson, Hesser, \& Smecker-Hane (1998) reveals that the overdensity is apparently dominated by a relatively young main sequence stellar population. We propose that this clump is the first detection of shell structure in a dwarf galaxy: such structure, despite being well observed in large galaxies, was not previously known in small systems such as dwarf galaxies. The recognition of shell structure in elliptical galaxies (e.g. Malin \& Carter (1980)) provided an important insight into understanding the interactions between a large galaxy and a smaller companion. Phase-wrapped shells in the haloes of large galaxies became obvious through the process of photographic amplification (Malin 1978) of images of elliptical galaxies (e.g. Malin, Quinn, \& Graham (1983)). Subsequent simulations by Hernquist \& Quinn $(1988,1989)$ indicated that a large galaxy disrupting a small companion will produce the observed structure, with some $(\sim 10 \%)$ of the material of the companion galaxy distributed in shells around the primary. The level of ordered structure in the shells reveals the type of interaction between the two objects: a direct collision will produce highly ordered, phase-wrapped shells around the primary galaxy, while a companion on a non-radial orbit will produce a confused set of shells. Observational evidence supports this process of shell formation. In particular, studies of the colour of the shells shows that they are generally bluer than the colour of the primary galaxy, reflecting the stellar population of the disrupted lower mass companion (Pence 1986; McGaugh \& Bothun 1990).

In the following section we describe our Fornax observations and the data reduction techniques. Section 3 outlines the analysis of the data and the results are discussed in section 4. The conclusions are presented in section 5 . 


\section{Observations and Data Reduction}

Initial deep images of the Fornax dwarf galaxy were derived by combining photographically amplified derivatives (Malin 1978; Bland-Hawthorn, Shopbell \& Malin 1993) from two deep plates taken with the UK Schmidt Telescope. The original exposures (J5462 and J8297) were made on hypersensitised Kodak IIIaJ emulsion with a Schott GG395 filter defining a broad passband from 395 to approximately 540nm. The shell-like structure is readily visible on the derivatives made from both plates and is undoubtedly real. In Fig. 1, the two photographically-amplified derivatives have been photographically combined to improve the signal-to-noise in the final image. The feature was further confirmed by inspecting the Digitised Sky Survey, and it is also present in the APM catalogue (Maddox et al. 1996). This prompted further analysis using CCD data.

CCD images were obtained with the Siding Spring Observatory 1 metre telescope using the Wide-Field Imager (WFI) in October/November 2002. WFI comprises eight $4096 \times 2048$ CCDs arranged in a $2 \times 4$ mosaic to give a total format of $8192 \times 8192$ pixels. This gives a field-of-view of $52 \times 52$ arcmin at a scale of $0.38^{\prime \prime}$ per pixel. The field centre was placed at the coordinates $\alpha=02^{h} 41^{m} 34.2^{s}, \delta=-34^{\circ} 48^{\prime} 06^{\prime \prime}$ (J2000.0), which lies approximately $31.8^{\prime}$ southeast from the centre of Fornax. Thus, the field includes the central region of Fornax. We recorded images in both $V$ and $I$ with exposure times of $6 \times 600$ s and $6 \times 480$ s, respectively, under good conditions $\left(\right.$ FWHM $\left.\approx 1.5^{\prime \prime}\right)$. These data form a small part of our large-scale photometric study of the Fornax dSph galaxy.

Image reduction was accomplished using standard IRAF routines. The bias images and overscan region allowed the pedestal current to be subtracted from all object and flat field exposures. Each object image was divided by the appropriate combined flat, accounting for variations in CCD pixel sensitivity. Fringing effects in the $I$-band are negligible.

After registering and combining the images for each filter, instrumental magnitudes were 
measured using the DAOPHOT (Stetson 1987) program within IRAF, which allows the point-spread function to be determined interactively. The full-width half-maximum values for the PSF were 4.8 pixels $\left(1.8^{\prime \prime}\right)$ and 3.5 pixels $\left(1.3^{\prime \prime}\right)$ in $V$ and $I$, respectively.

The magnitudes and corresponding colours were calibrated using Graham (1982) and Landolt (1992) standard fields observed across the CCDs of the WFI mosaic throughout the run. The extinction coefficients adopted were $k_{V}=0.16$ and $k_{I}=0.086$ from Sung \& Bessell (2000). As an example, the transformation equations for CCD 7 on the nights the data discussed here were obtained are:

$$
\begin{gathered}
V-v=24.26+0.006(V-I) \\
I-i=24.79-0.014(V-I)
\end{gathered}
$$

where $v$ and $i$ represent extinction corrected instrumental magnitudes using a photometry offset of 23.5. The rms scatter about these relations are 0.025 and 0.027 mag, respectively. The equations for CCD 8 are similar.

We present the results here for CCDs 7 and 8 which occupy the north-west quarter of the WFI mosaic. These CCDs contain the centre of Fornax and the overdense region as shown in Fig. 2. The photometric errors used are those returned by the DAOPHOT program.

\section{Results}

The first question to be addressed is whether the feature might be the result of differential extinction in the the Fornax field. We approached this question in two ways. First, we chose two regions, each $\sim 10^{\prime}$ in size. One was located near the centre of Fornax and the other centred in the vicinity of the feature. Using the extinction maps of Schlegel, 
Finkbeiner, \& Davis (1998), the difference in $E(B-V)$ between these two regions was investigated and found to be less than 0.01 mag. Minor changes in the positions of the regions do not alter this result. Second, we inspected the IRAS $100 \mu \mathrm{m}$ images of this part of the sky and found that there is no 'cirrus' structure in the vicinity of the Fornax feature. We conclude therefore that the feature is not a consequence of differential extinction.

We now consider the region of the feature in more detail. It is centred approximately $17^{\prime}$ (670 pc in projection) from the centre of Fornax at coordinates $\alpha=02^{h} 40^{m} 28.5^{s}$, $\delta=-34^{\circ} 42^{\prime} 33^{\prime \prime}$ (J2000.0). The physical characteristics were determined by convolving the $V$-band image with a circular Gaussian kernel using the IRAF subroutine gauss and a standard deviation of $\sigma=30$ pixels $\left(11.3^{\prime \prime}\right)$. This $\sigma$ value was chosen to merge small-scale structure within the feature, while allowing us to determine its overall dimensions. Defining a sampling resolution of $x_{\text {res }}=y_{\text {res }}=64$ pixels $\left(24^{\prime \prime}\right)$, we produced a contour image using the subroutine contour in the IRAF package. The feature is clearly detected in this convolved image. It is almost rectangular in shape with approximate dimensions $1.7^{\prime} \times 3.2^{\prime}$ $(68 \times 128$ parsecs at the distance of Fornax). The feature major axis has a position angle of $41^{\circ} \pm 2.5^{\circ}$ measured eastwards from north. This agrees closely with the position angle of the major axis of Fornax, which Irwin \& Hatzidimitriou (1995) give as $41^{\circ} \pm 1.0^{\circ}$. In other words, the major axis of the feature is parallel to the major axis of Fornax. The feature also lies very close to the minor axis of Fornax. According to Piatek et al. (2002), the proper motion of Fornax is also approximately in the direction of the galaxy's minor axis.

Using the IRAF routine polyphot, we next calculated the average surface brightness of a typical region at the same (ellipticity-corrected) distance from the centre of Fornax as the feature. This is $26.1 \pm 0.1 \mathrm{mag} / \operatorname{arcsec}^{2}$ in $V$. The feature, however, has an average surface brightness of $25.8 \pm 0.1 \mathrm{mag} / \operatorname{arcsec}^{2}$ in $V$, indicating an increased stellar density of approximately $30 \%$. The average surface brightness and the area of $1.7^{\prime} \times 3.2^{\prime}$, together 
with the background, then correspond to a total magnitude for the excess density of $m_{V}=16.6 \pm 0.6$. Assuming the stellar population of the overdensity is at the same distance as Fornax $\left((m-M)_{V}=20.80\right.$ for $E(B-V)=0.03$ (Mateo 1998)), the total absolute visual magnitude is $M_{V} \approx-4.2 \pm 0.6$ and the visual luminosity is $L_{V} \approx 4 \pm 2 \times 10^{3} L_{\odot}$. For comparison, the faintest of the five Fornax globular clusters, Fornax 1, has $M_{V}=-5.4$ and a limiting radius of $1.25^{\prime}$ (Mackey \& Gilmore 2003). This cluster is readily visible in the NW of Fig. 1 and the difference in size compared to the feature is apparent. Within the Galactic halo, globular clusters of comparable absolute magnitude to the feature include Pal $12(-4.5)$ and Pal $13(-3.7)$ (Harris 1996).

In Fig. 3 we show a colour-magnitude diagram (CMD) for Fornax using the two CCDs in the northwest quadrant of the WFI mosaic. As noted above (see also Fig. 2), these two CCDs cover the central parts of Fornax as well as the region of the overdensity. We were able to measure 5195 and 10312 stars, respectively, on these two CCDs. The high galactic latitude of Fornax means that contamination from foreground stars is minimal. The resulting CMD reaches down to the red clump at $V \approx 21$ with typical magnitude and colour errors less than 0.05 mag. We then compared the CMD for the approximately 100 stars in the overdense region with that for Fornax as a whole. This is also shown in Fig. 3 where the photometry for the stars in the region are shown overlaid on the Fornax CMD. No obvious differences in the CMD distributions are immediately apparent but this is not surprising given that approximately two in every three stars in the overdense region are members of the Fornax field rather than of the overdense region. A deeper dataset is needed for further analysis.

Fortunately, the deep survey of Fornax conducted by Stetson, Hesser, \& Smecker-Hane (1998) is available, providing a basis for additional analysis of the stellar population of the overdensity. The Stetson, Hesser, \& Smecker-Hane (1998) data were obtained using the 
Cerro Tololo $1.5 \mathrm{~m}$ telescope, and a full description of their data reduction techniques can be found in their paper. Whereas our data ends at the red clump of Fornax at $V \approx 21$, the

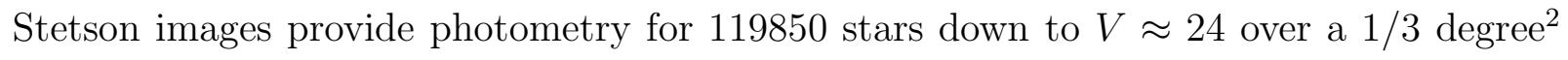
field, revealing, inter alia, a relatively young main sequence. The overdense region lies at the edge of the Stetson field.

Figure 4 shows the CMD for the entire Stetson dataset with the stars from the overdense region superposed. There are 327 stars in this region and the excess above the expected star numbers at this (ellipticity-corrected) radius is approximately 40\%; a value in reasonable agreement with that derived from the WFI data. It is immediately apparent from Fig. 4 that there is a high concentration of relatively young main sequence stars in the overdense region. We have confirmed this occurrence in two ways.

First, we selected from the Stetson dataset those stars in the main sequence CMD region outlined in Fig. 4. The spatial distribution of these stars is shown in Fig. 5. The overdense region stands out prominently in the bottom left of the figure. Note that we have excluded the inner regions of Fornax in making this plot as there are potential substantial inter-field completeness variations for stars in this part of the CMD in the central regions of the dwarf (Stetson, Hesser, \& Smecker-Hane 1998). The prominence of the overdensity when using relatively blue stars also explains why the feature is better seen on blue plates. We have not conducted a statistical analysis of this map, but it appears that the overdense region is the largest fluctuation. A more detailed analysis, which allows for variable incompleteness, may reveal additional overdensities. Stetson, Hesser, \& Smecker-Hane (1998) did note, however, that the younger main sequence (blue) stars, defined as those with $18.5 \leq(B+R) / 2 \leq 22$ (essentially equivalent limits apply for $B$ for these stars which have $B-R \approx 0$ ) possess "a clearly flattened distribution on the sky with the long axis in the $\mathrm{E}-\mathrm{W}$ direction and an axial ratio of 3 or 4 to 1 ". This distribution is evident in Fig. 5 
as the excess density of points to the East and West of the excluded region; see also Fig. 12 of Stetson, Hesser, \& Smecker-Hane (1998).

Second, in order to make an unbiased assessment of the stellar population belonging to the overdense region, we have endeavoured to generate a background-subtracted CMD for the region. Two complimentary methods were constructed for this CMD subtraction process. For the first, we defined a CMD density function $\Phi$, generated by dividing the Fornax CMD into a grid of $34 \times 34$ cells in colour and magnitude and then counting the stars per cell. The cells were equally spaced, extending through the domain $-0.4 \leq(B-R) \leq 3.0$ and the range $19.15 \leq B \leq 23.95$. Each cell covered an area $0.1 \times 0.15 \mathrm{mag}^{2}$. An estimate of the CMD for the overdense region was then determined by subtracting the 'average' Fornax CMD density function $\Phi_{\text {Fornax }}$ from that for the overdense region. That is,

$$
\Phi_{\text {feature }}=\Phi_{\text {region }}-\Phi_{\text {Fornax }}
$$

To avoid the introduction of errors in the function $\Phi_{\text {Fornax }}$ by any possible radial change in the stellar population of Fornax, we chose stars only at the same (ellipticity-corrected) radius from the centre of Fornax as the overdense region. In other words, we used an annulus with the same ellipticity-corrected radius and width as the overdense region to select the Fornax stars for our average CMD for the dSph. The sample for $\Phi_{\text {Fornax }}$ was then normalised by reducing the area of the annulus to be the same as that of the overdense region. This gave the number of stars in an average region at the same radius as the overdense region as $\sim 226$, while the Stetson, Hesser, \& Smecker-Hane (1998) data for the overdense region contains 327 stars. This leads to a further estimate of the feature as a $\sim 45 \%$ overdensity, again consistent with the previous estimates above.

We then calculated the difference between $\Phi_{\text {region }}$ and $\Phi_{\text {Fornax }}$, and the resulting CMD density function for the overdense region is shown in Fig. 6. The greyscale shown ranges 
from zero (white, indicating no difference between the overdense region and Fornax) to 500 (black, indicating a difference of 500 stars $/ \mathrm{mag}^{2}$ at that location in the CMD) so that the darker regions in Fig. 6 indicate higher levels of confidence in the presence of overdensity members at that colour-magnitude value. Once again the CMD of the feature appears dominated by a relatively young main sequence population, consisting of approximately 80 stars. There is also some indication of the presence of red clump and red giant branch stars.

The second subtraction method used the 'average' Fornax field CMD defined above. We constructed a series of field CMDs by using a Monte Carlo alogrithm applied the density function, $\Phi_{\text {Fornax }}$. Each field CMD contained 226 stars, the average number of stars in a region with the same area and distance from the Fornax centre as the overdensity. We then subtracted each of these trial field CMDs by 'cancelling out' the nearest star in the CMD of the overdense region. A typical outcome from this process is shown in Fig. 7. In particular, we find that the occurrence of stars in the region $B \sim 22.5-23.5$ and $(B-R) \sim 0.3$ in the subtracted CMDs is a robust result of this process.

To estimate the age of the stars in the overdense region we have used isochrones calculated from the Yonsei-Yale web-based release (Yi et al. 2001; Kim et al. 2002). Shown in Figs. 6 and 7 are isochrones for ages of 1,2 and 3 Gyr and an assumed metallicity of $[\mathrm{Fe} / \mathrm{H}]=-1.0$. Given that the data are increasingly incomplete below $\mathrm{B} \approx 23.5$ (Stetson, Hesser, \& Smecker-Hane 1998) ${ }^{1}$, the isochrones in Figs. 6 and 7 suggest the overdense region

\footnotetext{
${ }^{1}$ Stetson, Hesser, \& Smecker-Hane (1998) actually state that they regard the data as 'essentially complete' to well below $[V=(B+R) / 2]$ magnitude 23. For main sequence stars with $B-R \approx 0.4, V=23$ corresponds to $B=23.2$ from which we assumed that the data are increasingly incomplete below $B \approx 23.5$. It is also worth noting, as Figure 2 of Stetson, Hesser, \& Smecker-Hane (1998) illustrates, that the region of the overdensity received less total exposure time than the central regions of the dwarf galaxy.
} 
is dominated by a stellar population with an age of $\sim 2$ Gyr for this metallicity. However, the limiting magnitude of the Stetson, Hesser, \& Smecker-Hane (1998) data in this region makes it difficult to decide whether the overdense region also contains an older population. The metallicity assumed was chosen on the basis of the fit to the CMD, and we note that Tolstoy et al. (2001) give the mean metallicity of Fornax as $[\mathrm{Fe} / \mathrm{H}] \approx-1.0$. Tolstoy et al. (2003) argue for an age-metallicity relation in Fornax and assign an age of $\sim 2 \mathrm{Gyr}$ to the Fornax giant M21 whose iron abundance, measured from a UVES high dispersion spectrum, is $[\mathrm{Fe} / \mathrm{H}]=-0.7$. However, the use of higher metallicity isochrones produces a less satisfactory agreement in colour at the main sequence, and does not substantially alter the age estimate. Deeper imaging specific to the overdense region would aid substantially in constraining the age (or age range) of its stellar population.

\section{Discussion}

We turn now to the interpretation of the overdense region. Our current understanding of dSph galaxies indicates they are complex systems, with star formation histories extending back to the earliest times (Mateo (1998) and references therein). These systems may be the darkest in the universe, with mass-to-light ratios ranging from $M / L \sim 5$ for the higher luminosity dwarfs such as Fornax and Sculptor, to $M / L \sim 100$ for systems such as Draco and Ursa Minor (Mateo 1998). According to CDM theory, the presence of such a dominant dark halo should inhibit the formation of significant substructure, barring strong encounters with objects of a comparable or higher mass (e.g. the Sagittarius and NGC 205 dwarf galaxies are being tidally stripped by their host galaxies). Therefore, we do not expect the feature to be intrinsic structure in the dSph. However, Kleyna et al. (2003) have recently argued that the observed morphological substructure in the Ursa Minor dSph could be a primordial artifact. From their models, the primary requirements for a dynamically cold 
clump to survive are that the dark matter potential possess a constant density core, and that core size be substantially larger than the orbit: substructure is rapidly destroyed in the cuspy dark matter potential predicted by CDM or for orbits larger than the core radius (Kleyna et al. 2003). Nevertheless, the Fornax overdense region is unlikely to be a primordial survivor (i.e. formed at early epochs) as it contains stars as young as a few Gyr (cf. Figs. 6 and 7).

An initially plausible interpretation of the overdense region is that it is a consequence of tidal interaction between Fornax and the Milky Way Galaxy. For example, recent studies of the Ursa Minor dSph (UMi) by Martínez-Delgado et al. (2001); Bellazzini et al. (2002); Palma et al. (2003) and Kleyna et al. (2003) have revealed a strongly disturbed structure for this dSph. This is frequently interpreted as the result of tidal interactions with the Galaxy. However, this is unlikely to be the origin of the overdense region in Fornax, for two reasons. First, the galactocentric distance and mass of Fornax are considerably larger than those of Ursa Minor and thus Fornax is subject to substantially lower tidal forces. Second, and more compelling, is the fact that the overdense region has a stellar population that appears to be distinct from that for the bulk of Fornax. If the feature had a tidal origin, the expectation would be that the feature population should be the same as that for the dwarf as a whole, and this is not the case.

Another possible interpretation is that the overdense region represents the remnant of a disrupted stellar cluster, which presumably was a few Gyr in age. As noted above, the Kleyna et al. (2003) models illustrate that a clump formed in this way could survive if two main criteria are fulfilled. Firstly, the distribution of the dark matter (DM) in Fornax must have an approximately uniform density core (that is, it differs from the standard CDM prediction). If the core is cusped, then any coherent structure is disrupted within a few orbital periods. We estimate the orbital period of the clump at this radius to be $\sim 10^{8}$ years 
using the Fornax central density and core radius values from Mateo (1998), considerably less than the inferred age. Secondly, the core radius of the DM distribution should be at least comparable with the distance of the clump from the centre of Fornax. This latter point is possible; the clump lies in projection at about $1.5 \mathrm{r}_{\text {core,stars }}$ and the available velocity dispersion results suggest that the dominant dark mass probably has a more extended distribution than the light (Mateo et al. 1991). The signature of this interpretation would be that the clump possesses a single age population, and that it is kinematically cold with a velocity dispersion substantially less than that of the dwarf. It would a considerable observational challenge to determine if the overdensity has such as small velocity dispersion. However, with deeper imaging data it should be possible to determine if the overdensity is dominated by a single age population, as would be expected if it is a disrupted cluster, or contains a mix of populations. Such deeper imaging observations are planned. We note, however, that while on-going star formation has certainly occurred in Fornax, there is no evidence for any star clusters in this dwarf other than the five well known globular clusters, which all have ages comparable to the globular clusters of the Galactic halo (Buonanno et al. 1998, 1999) and which all show no signs of disruption (Mackey \& Gilmore 2003; Rodgers \& Roberts 1994). Therefore, it seems unlikely that the clump is the remnant of a disrupted $\sim 2$ Gyr old cluster. We further note that there are no stellar aggregates (bound or unbound) in any other dSph that have ages of a few Gyr. Such aggregates that do exist are either very old (such as the Fornax globular clusters or the Ursa Minor kinematically cold clump) or very young. For example, the Phoenix dSph/dIrr contains an apparent association of young (age 100 Myr) stars (Martínez-Delgado, Gallart, \& Aparicio 1999). This young association is located towards the centre of the Phoenix dwarf, in contrast to the Fornax feature which is situated significantly beyond the core radius of the dwarf.

Our preferred interpretation is that the overdense region represents a 'shell' analogous to those seen around many E galaxies: the shells arise from orbit phase-wrapping of the 
debris of a low-mass companion tidally disrupted by a more massive galaxy (e.g. Hernquist \& Quinn $(1988,1989))$. Such features are relatively common in more luminous systems, with about $\sim 10 \%$ of all E galaxies showing features of this nature (Hernquist \& Quinn 1988), however such structure has not been previously seen in dE or dSph galaxies. Our shell interpretation is driven by the location and appearance of the Fornax overdense region - the location on the minor axis, the elongation parallel to the major axis, and the relatively 'sharp-edged' nature of the overdense region are all characteristic of shell features. Shells around more luminous E galaxies are also generally bluer than the primary galaxy (Pence 1986; McGaugh \& Bothun 1990) and that is clearly also the case here. Simulations by Hernquist \& Quinn (1989) predict approximately 10\% of the companion mass is distributed as shells around the primary, depending on the mass ratio and collision parameters of the two objects. If we assume that approximately $1-10 \%$ of the mass contained in the shells is visible in this clump, we obtain a mass of $\sim 10^{6}-10^{7} \mathrm{M}_{\odot}$ for the companion object. This is comparable to the smallest dwarf galaxies of the Local Group.

We propose then that a relatively gas-rich low mass dwarf galaxy merged with Fornax approximately 2 Gyr ago. Tidal stirring of gas from the companion produced a new population of stars, which, together with the remnant population of the disrupted system, are distributed as shell structure throughout the Fornax dSph galaxy. Modelling of such a process may well provide insight as to the location and surface density of other possible shells to guide further observations. It may also indicate how retention of gas from such a merger process may have led to the unusual distribution of the youngest Fornax stars as outlined by Stetson, Hesser, \& Smecker-Hane (1998). 


\section{Conclusion}

We have presented photometric data for a stellar clump located $\sim 17^{\prime}$ from the centre of the Fornax dwarf spheriodal galaxy. The clump is approximately $68 \times 128 \mathrm{pc}$ in size and is $\sim 40 \%$ more dense than the surrounding regions. It is located on the Fornax minor axis and is elongated in the direction of the dSph's major axis. By removing the Fornax field contamination through a CMD density function analysis, we have shown that the clump appears dominated by a stellar population approximately 2 Gyr in age. Our interpretation of this clump is that it is a shell feature analogous to those frequently seen around more luminous E galaxies. As such, it would represent the first detection of shell structure in a dwarf galaxy. In this interpretation we propose that a smaller gas-rich system merged with Fornax in the relatively recent past ( $\sim 2$ Gyr ago) and that the shell is a consequence of this merger. Models of such a merger, kinematic studies of the shell stars, and further deep imaging of the region surrounding this dSph to constrain any additional shell structure, are all required to investigate this hypothesis more thoroughly. It may be that in many cases the comparitively recent star formation seen in some dSph and dE galaxies is also the result of the infall of lower mass, relatively gas-rich systems.

The authors are grateful to Peter Stetson for making his Fornax data available for this study. Matthew Coleman acknowledges the financial support provided by an Australian Postgraduate Award. The authors are also grateful to the referee, Dr. Antonio Aparicio, for his helpful comments on the original manuscript. 


\section{REFERENCES}

Bellazzini, M., Ferraro, F. R., Origlia, L., Pancino, E., Monaco, L., \& Oliva, E. 2002, AJ, 124,3222

Bland-Hawthorn, J., Shopbell, P. L., \& Malin, D. F. 1993, AJ, 106, 2154

Bouchard, A., Carignan, C., Mashchenko, S. 2003, AJ, in press (astro-ph/0306218)

Buonanno, R., Corsi, C. E., Zinn, R., Fusi Pecci, F., Hardy, E., \& Suntzeff, N. B. 1998, ApJ, 501, L33

Buonanno, R., Corsi, C. E., Castellani, M., Marconi, G., Fusi Pecci, F., \& Zinn, R. 1999, AJ, 118, 1671

Carignan, C., Beaulieu, S., Côté, S., Demers, S., \& Mateo, M. 1998, AJ, 116, 1690

Eskridge, P. B. 1988, AJ, 96, 1614

Graham, J. A. 1982, PASP, 94, 244

Harris, W. E. 1996, AJ, 112, 1487

Hernquist, L. \& Quinn, P. J. 1988, ApJ, 331, 682

Hernquist, L. \& Quinn, P. J. 1989, ApJ, 342, 1

Hodge, P. W. 1961, AJ, 66, 249

Irwin, M. \& Hatzidimitriou, D. 1995, MNRAS, 277, 1354

Kim, Y., Demarque, P., Yi, S. K., \& Alexander, D. R. 2002, ApJS, 143, 499

Kleyna, J. T., Wilkinson, M. I., Gilmore, G., \& Evans, N. W. 2003, ApJ, 588, L21

Landolt, A. U. 1992, AJ, 104, 340 
Mackey, A. D., \& Gilmore, G. F. 2003, MNRAS, 340, 175

Maddox, S. J., Efstathiou, G., Sutherland, W. J., \& Loveday, J. 1996, MNRAS, 283, 1227

Malin, D. F. 1978, Nature, 276, 591

Malin, D. F. \& Carter, D. 1980, Nature, 285, 643

Malin, D. F.\& Carter, D. 1983, ApJ, 274, 534

Malin, D. F., Quinn, P. J., \& Graham, J. A. 1983, ApJ, 272, L5

Martínez-Delgado, D., Gallart, C., \& Aparicio, A. 1999, AJ, 118, 862

Martínez-Delgado, D., Alonso-García, J., Aparicio, A., \& Gómez-Flechoso, M. A. 2001, ApJ, 549, L63

Mateo, M. 1998, ARA\&A, 36, 435

Mateo, M., Olszewski, E., Welch, D. L., Fischer, P., \& Kunkel, W. 1991, AJ, 102, 914

Mayer, L., Moore, B., Quinn, T., Governato, F., \& Stadel, J. 2002, MNRAS, 336, 119

McGaugh, S. S. \& Bothun, G. D. 1990, AJ, 100, 1073

Moore, B., Ghigna, S., Governato, F., Lake, G., Quinn, T., Stadel, J., \& Tozzi, P. 1999, ApJ, 524, L19

Olszewski, E. W., \& Aaronson, M. 1985, AJ, 90, 2221

Palma, C., Majewski, S. R., Siegel, M. H., Patterson, R. J., Ostheimer, J. C., \& Link, R. 2003, AJ, 125, 1352

Peacock, J. A. 1999, Cosmological Physics. (Cambridge: Cambridge University Press) 
Peebles, P. J. E. 1993, Principles of Physical Cosmology.(Princeton: Princeton University Press)

Pence, W. D. 1986, ApJ, 310, 597

Piatek, S., Pryor, C., Olszewski, E. W., Harris, H. C., Mateo, M., Minniti, D., Monet, D. G., Morrison, H., \& Tinney, C. G. 2002, AJ, 124, 3198

Rodgers, A. W., \& Roberts, W. H. 1994, AJ, 107, 1737

Saviane, I., Held, E. V., \& Bertelli, G. 2000, A\&A, 355, 56

Schlegel, D. J., Finkbeiner, D. P., \& Davis, M. 1998, ApJ, 500, 525

Shetrone, M. D., Côté, P., \& Sargent, W. L. W. 2001, ApJ, 548, 592

Stetson, P. B. 1987, PASP, 99, 191

Stetson, P. B., Hesser, J. E., \& Smecker-Hane, T. A. 1998, PASP, 110, 533

Sung, H. \& Bessell, M. S. 2000, Publications of the Astronomical Society of Australia , 17, 244

Tolstoy, E., Irwin, M. J., Cole, A. A., Pasquini, L., Gilmozzi, R., \& Gallagher, J. S. 2001, MNRAS, 327, 918

Tolstoy, E., Venn, K. A., Shetrone, M., Primas, F., Hill, V., Kaufer, A., \& Szeifert, T. 2003, AJ, 125, 707

Tosi, M. 2003, Ap\&SS, 284, 651

Walcher, C. J., Fried, J. W., Burkert, A., \& Klessen, R. S. 2003, A\&A, 406, 847

Weldrake, D. T. F., de Blok, W. J. G., \& Walter, F. 2003, MNRAS, 340, 12 
Yi, S., Demarque, P., Kim, Y., Lee, Y., Ree, C. H., Lejeune, T., \& Barnes, S. 2001, ApJS, 136,417 


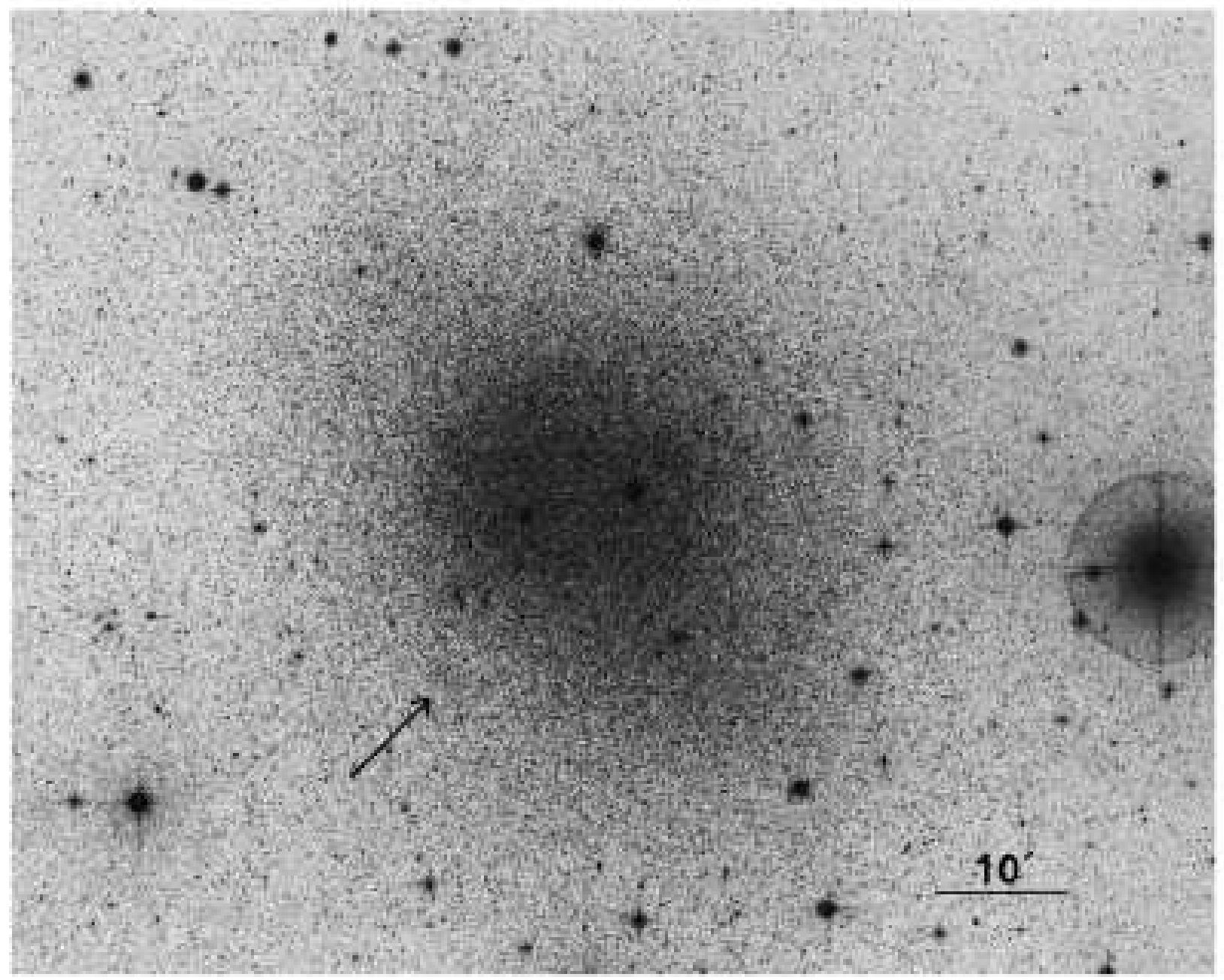

Fig. 1.- A deep image of Fornax from a combination of photographically-amplified derivatives from two UK Schmidt telescope IIIaJ plates. The feature of interest is towards the lower-left of the image and is indicated by the arrow. In this image North is up and East is to the left. 


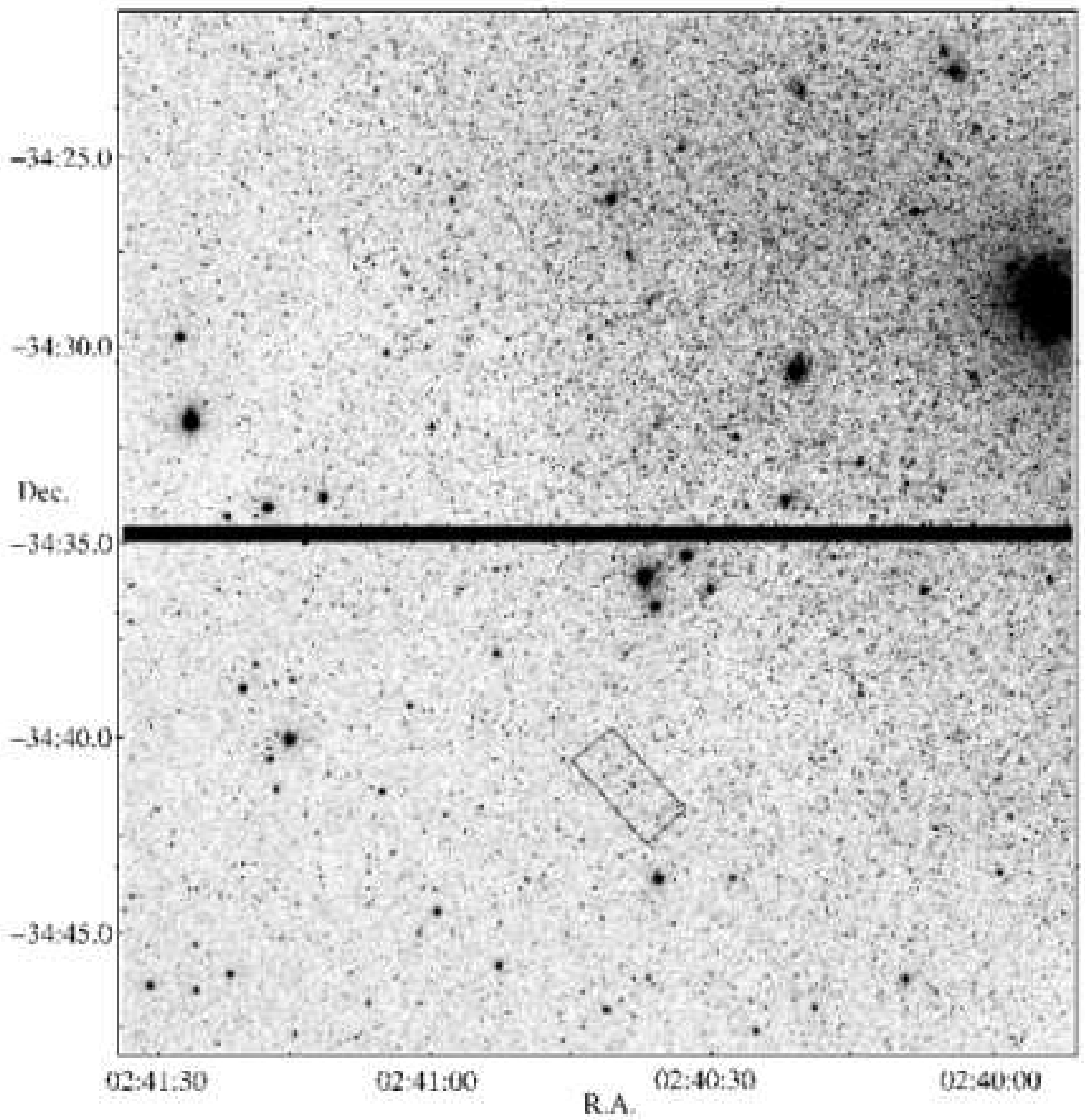

Fig. 2.- A $V$-band image of Fornax from the Wide-Field Imager (WFI) on the SSO $1 \mathrm{~m}$ telescope. Shown are CCDs 7 (bottom) and 8 (top), which cover an area $26^{\prime} \times 26^{\prime}$ on the sky. The overdense feature is the region outlined by the rectangle near the centre of CCD 7. As for Fig. 1, North is up and East is to the left. 


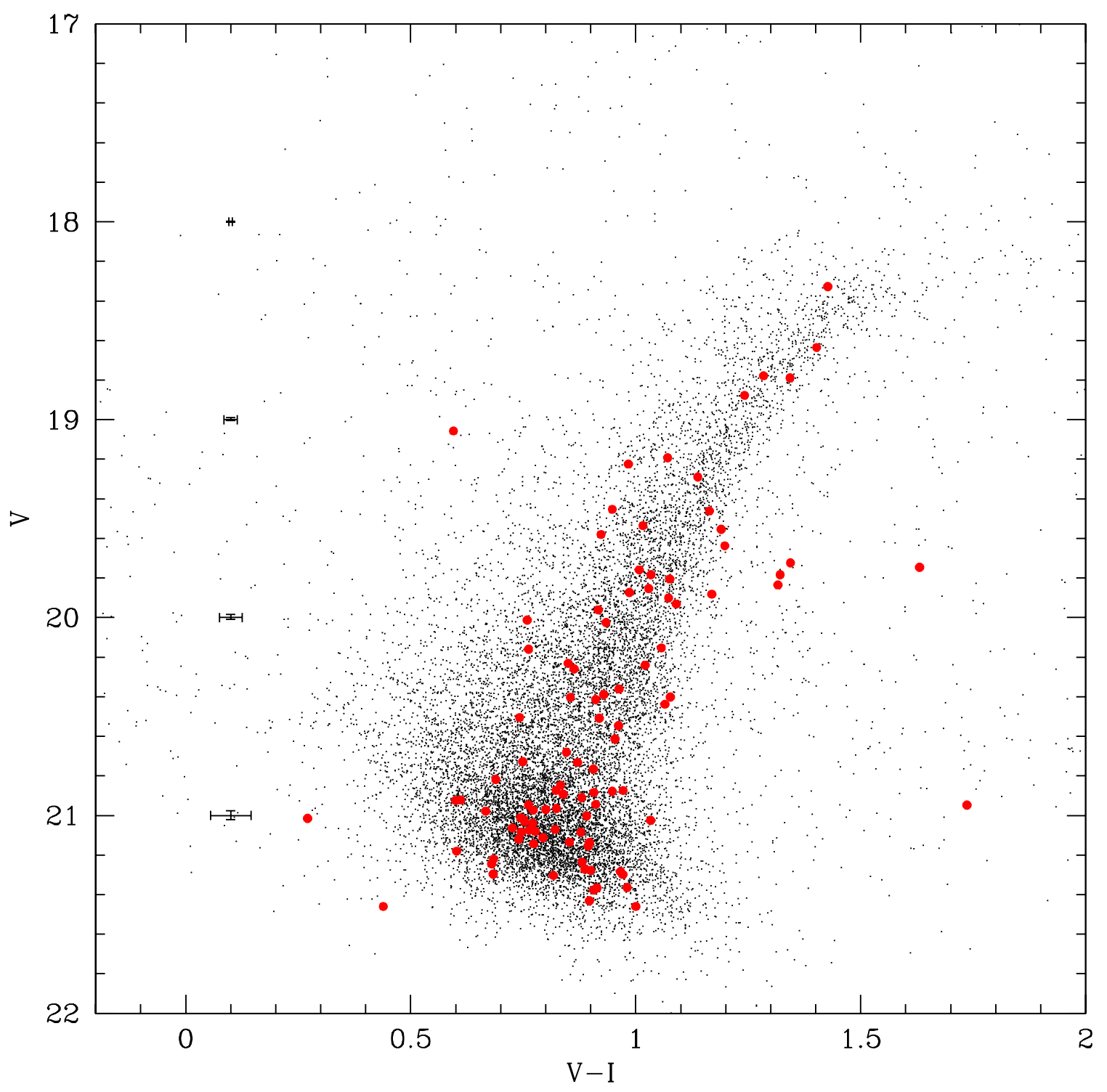

Fig. 3.- A colour-magnitude diagram for Fornax from WFI CCDs 7 and 8 that reaches the red clump at $V \approx 21$. Overlaid is the photometry for the stars in the region outlined in Fig. 2 . 


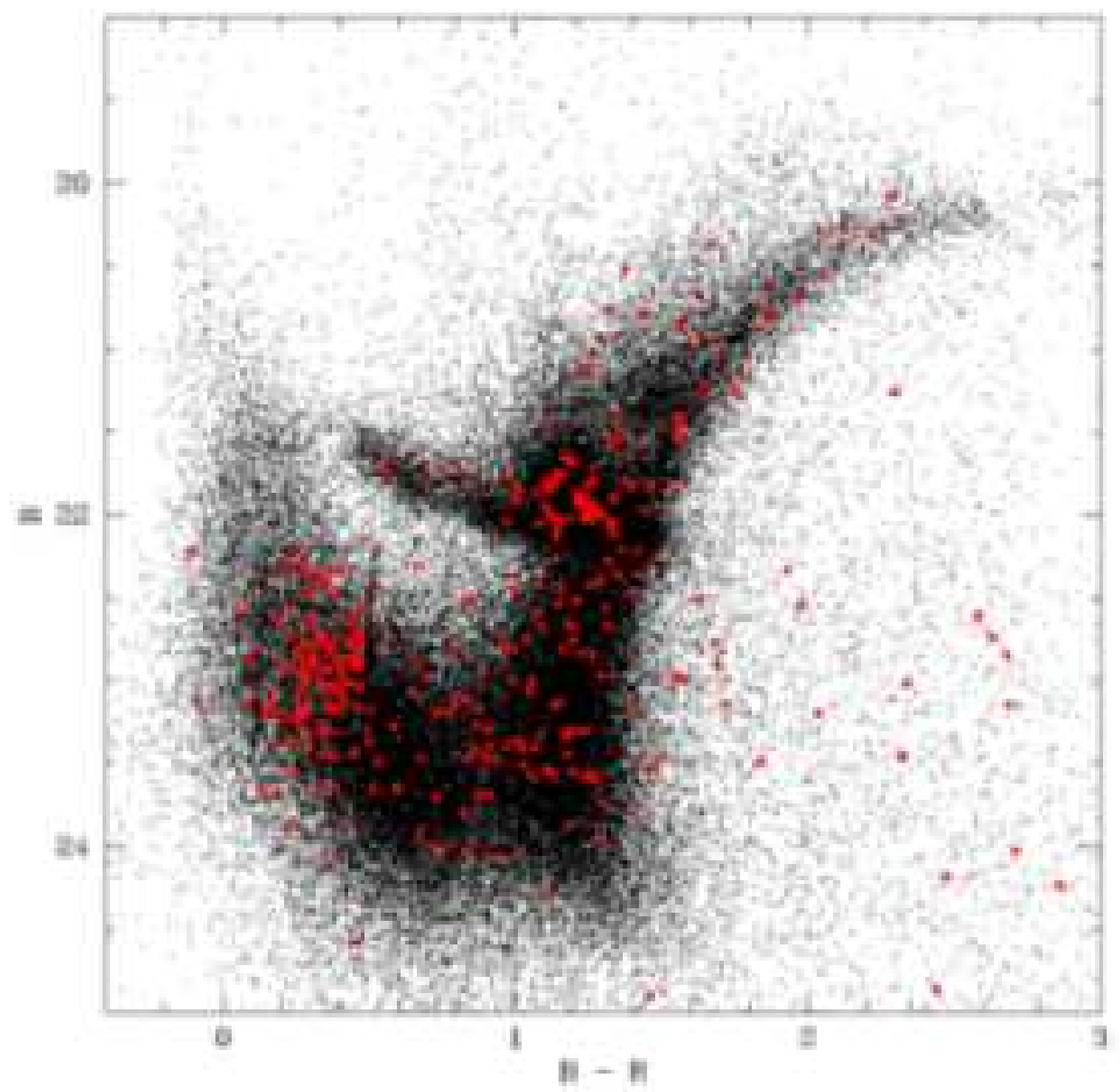

Fig. 4.- A colour-magnitude diagram for Fornax from the dataset of Stetson, Hesser, \& Smecker-Hane (1998). Overlaid are the points for the stars that lie within the boundaries of the overdense region. A selection box for main sequence stars is outlined. 


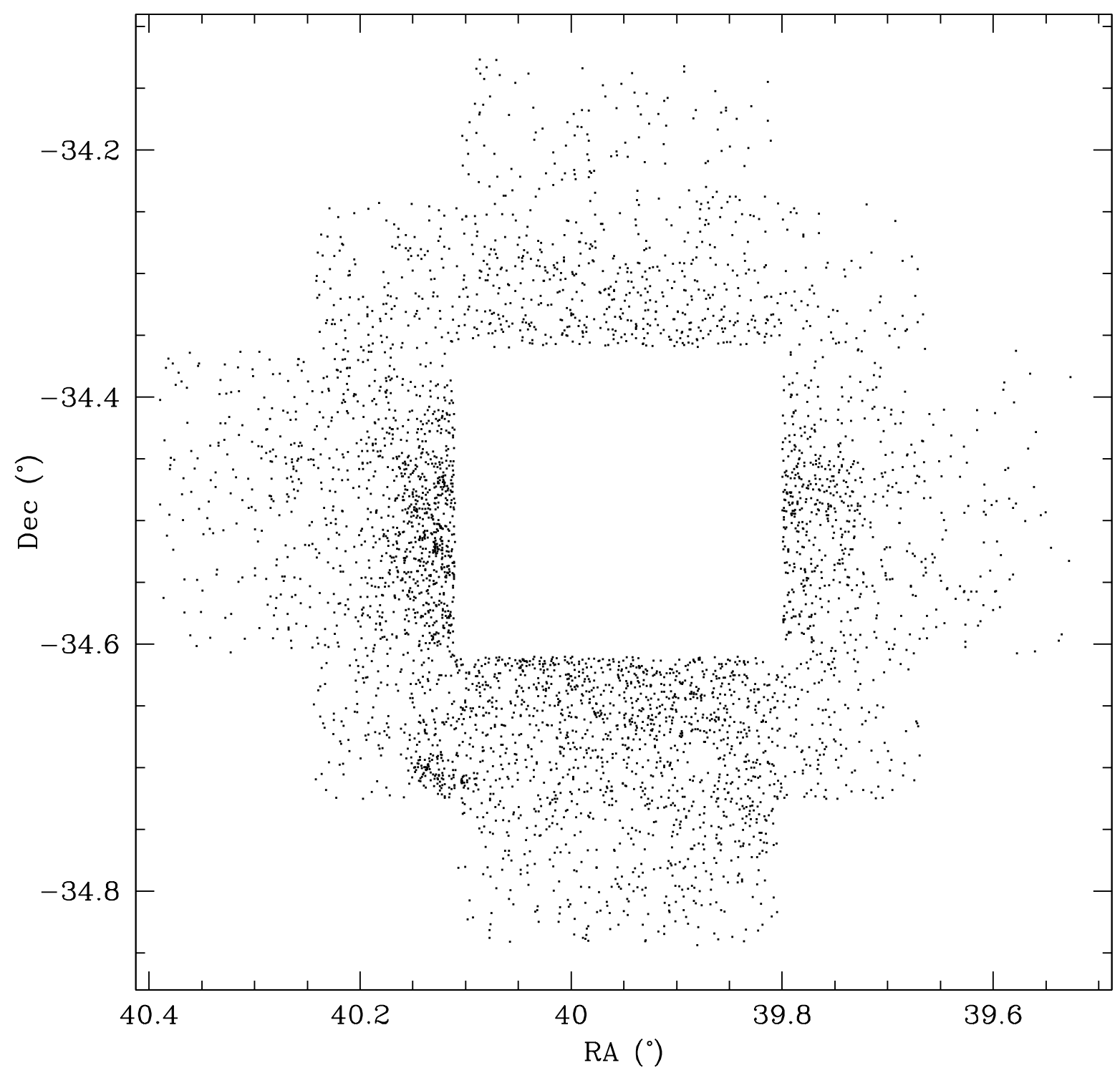

Fig. 5.- The spatial distribution from the Stetson, Hesser, \& Smecker-Hane (1998) dataset of main sequence stars in the CMD selection box shown in Fig. 4. The overdense region is clearly visible in the lower left of the figure, which has the same orientation as Figs. 1 and 2. The central regions of Fornax experience substantial inter-field completeness variations at the selected colours and magnitudes, and have been excluded. 


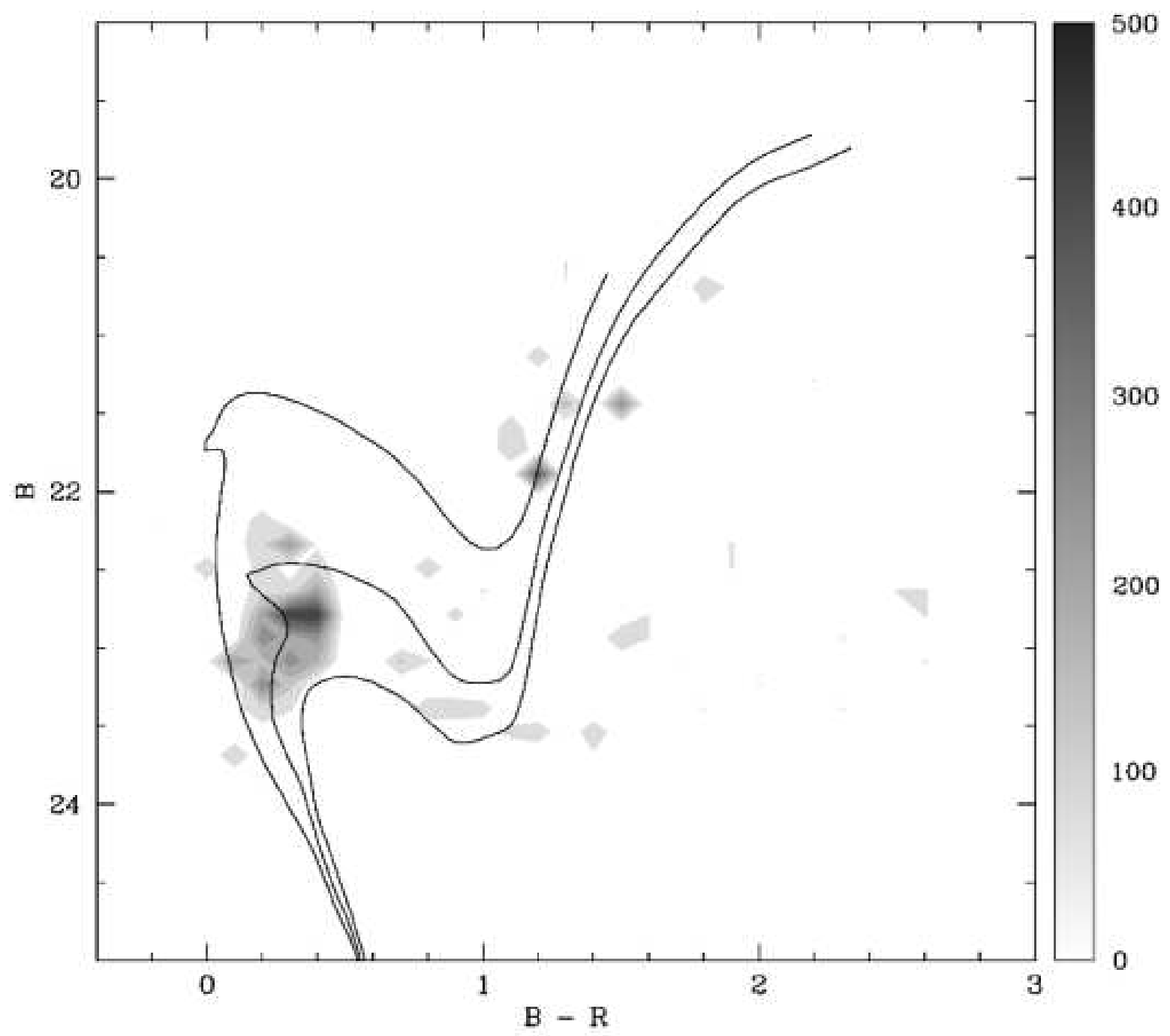

Fig. 6.- The background-field-subtracted CMD density function for the overdense region, $\Phi_{\text {feature }}$. Overplotted are Yonsei-Yale isochrones for ages of 1, 2 and 3 Gyr, and an assumed metallicity $[\mathrm{Fe} / \mathrm{H}]=-1.0$. The density column at the right hand side defines the stellar density in the CMD in stars $/ \mathrm{mag}^{2}$. The largest arrangement centred at $B \sim 23,(B-R) \sim 0.3$ corresponds to approximately 80 stars. 


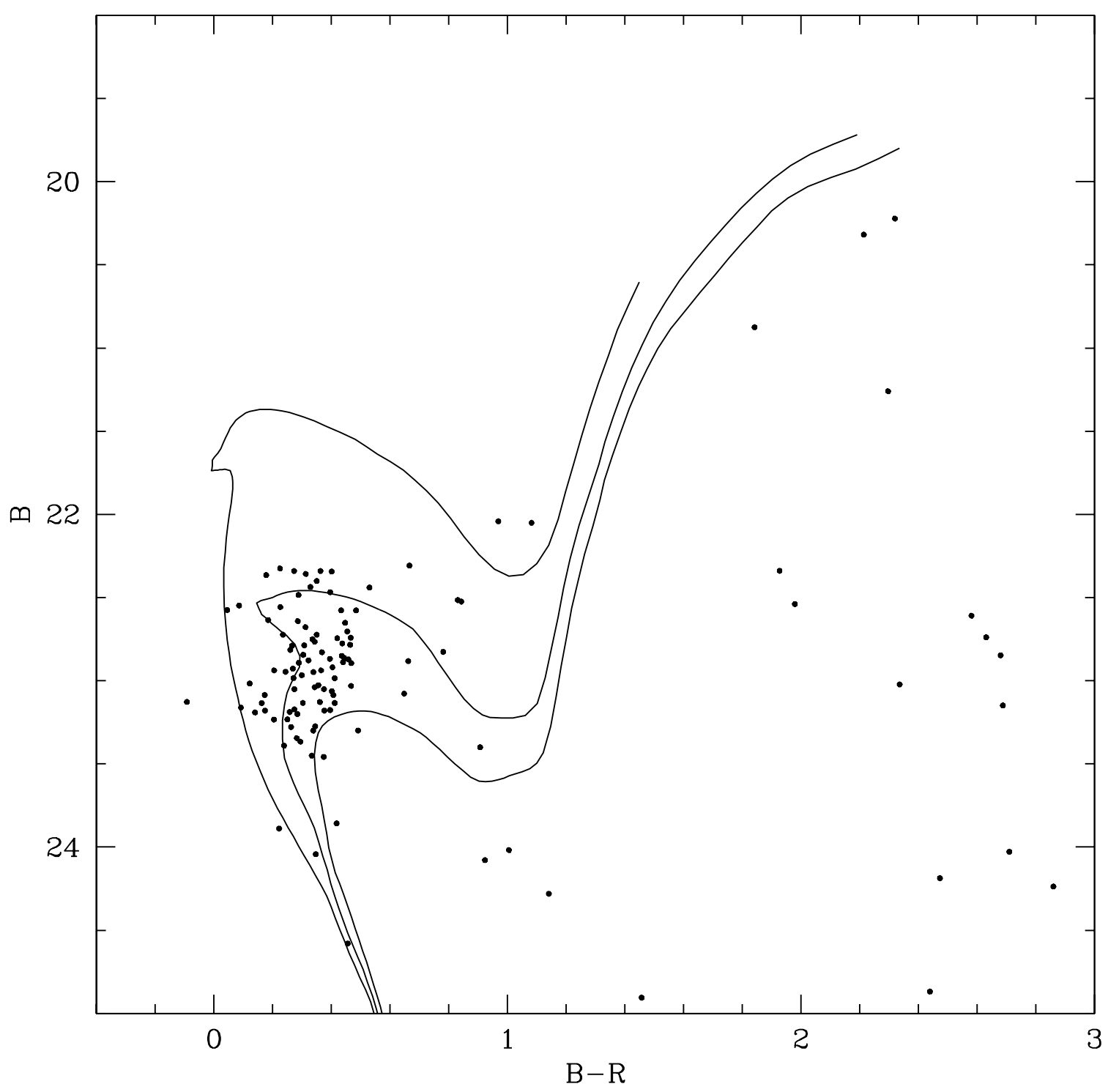

Fig. 7.- Subtracted CMD for the overdense region constructed using a Monte Carlo subtraction technique. Overplotted are Yonsei-Yale isochrones for ages of 1, 2 and 3 Gyr, and an assumed metallicity $[\mathrm{Fe} / \mathrm{H}]=-1.0$. 